\title{
A HERMITE PSEUDO-SPECTRAL METHOD FOR SOLVING SYSTEMS OF GROSS-PITAEVSKII EQUATIONS *
}

\author{
RADA M. WEISHÄUPL ${ }^{\dagger}$, CHRISTIAN SCHMEISER ${ }^{\ddagger}$, PETER A. MARKOWICH ${ }^{\S}$, AND \\ JUAN PABLO BORGNA
}

\begin{abstract}
We propose and analyze discretization methods for solving finite systems of nonlinearly coupled Schrödinger equations, which arise as an asymptotic limit of the three-dimensional Gross-Pitaevskii equation with strongly anisotropic potential. A pseudo-spectral method with Hermite basis functions combined with a Crank-Nicolson type method is introduced. Numerical experiments are presented, including a comparison with an alternative discretization approach.
\end{abstract}

Key words. Gross-Pitaevskii equation, spectral decomposition, Fourier expansion, Hermite polynomials

AMS subject classifications. 33C45, 35Q55, 65M70

\section{Introduction}

The first experimental realization of a Bose-Einstein condensate (BEC), achieved in 1995 for atomic gases [1], stimulated numerous experimental and theoretical studies in the succeeding years. Crucial ingredients in producing a BEC are laser cooling and trapping of atoms. A wide experimental interest is dedicated to BEC in highly anisotropic configurations. In this context it can be observed that by changing the shape of the trapping potential, it is possible to produce cigar- or disk-shaped BEC [13].

Below the critical condensation temperature the evolution of BEC is well described by the Gross-Pitaevskii equation (GPE), which is a nonlinear Schrödinger equation with harmonic potential modeling the magnetic trap and a cubic nonlinearity describing two particle bosonic interaction $[2,10,14,16]$. The solution $\psi=\psi(t, x, z)$ is the macroscopic wave function of the condensate, defined for $x \in \mathbb{R}^{e}, z \in \mathbb{R}^{d}, e+d=3$, and $t>0$.

$$
i \hbar \psi_{t}=-\frac{\hbar^{2}}{2 m} \Delta \psi+\frac{m}{2}\left(\omega_{x}^{2}|x|^{2}+\omega_{z}^{2}|z|^{2}\right) \psi+N_{a} g|\psi|^{2} \psi,
$$

where $m$ is the atomic mass, $\hbar$ is the Planck constant, $N_{a}$ is the number of atoms in the condensate, and $\omega_{x}, \omega_{z}$ are the trap frequencies in the $x$ - and $z$-directions, respectively. The parameter $g$ describes the interaction between the atoms in the condensate and has the form $g=\hbar^{2} a / m$, where $a$ is the scattering length, positive for repulsive interactions and negative for attractive interactions. Characteristic lengths of the condensate in the $x$ - and $z$-directions are $a_{x}=\sqrt{\hbar /\left(m \omega_{x}\right)}$ and $a_{z}=\sqrt{\hbar /\left(m \omega_{z}\right)}$, respectively.

*Received: December 16, 2006; accepted (in revised version): February 20, 2007. Communicated by Shi Jin.

${ }^{\dagger}$ Faculty of Mathematics, University of Vienna, Nordbergstr. 15/ C717 1090 Vienna, Austria (rada.weishaeupl@univie.ac.at).

${ }^{\ddagger}$ Faculty of Mathematics, University of Vienna, Nordbergstr. 15/ C707 1090 Vienna, Austria (christian.schmeiser@univie.ac.at).

§Institut für Mathematik, Universität Wien, Nordbergstr. 15, 1090 Vienna, Austria.

IDepartamento de Matematica, Universidad de Buenos Aires, Argentina. 
We introduce the scaling

$$
x \rightarrow a_{x} x, \quad z \rightarrow a_{z} z, \quad t \rightarrow \frac{t}{\omega_{x}}, \quad \psi \rightarrow \frac{\psi}{\sqrt{a_{x}^{e} a_{z}^{d}}},
$$

and introduce the dimensionless parameters

$$
\varepsilon:=\sqrt{\frac{\omega_{x}}{\omega_{z}}} \quad \text { and } \quad \gamma:=\frac{N_{a} a}{a_{z}^{d} a_{x}^{e-2}} .
$$

We assume a highly anisotropic confinement, i.e. $\varepsilon \ll 1$, and weak coupling, i.e. $\gamma=$ $\mathcal{O}(1)$.

The initial value problem for the scaled version of (1.1) then becomes

$$
\begin{gathered}
i \partial_{t} \psi=H^{\perp} \psi+\frac{1}{\varepsilon^{2}} H \psi+\gamma|\psi|^{2} \psi, \\
\psi(t=0, x, z)=\psi_{I}(x, z), \quad x \in \mathbb{R}^{e}, z \in \mathbb{R}^{d} .
\end{gathered}
$$

Here $H^{\perp}=-\frac{1}{2} \Delta_{x}+\frac{|x|^{2}}{2}$ and $H=-\frac{1}{2} \Delta_{z}+\frac{|z|^{2}}{2}$ are the Hamiltonians of the harmonic oscillators in $x$ - and $z$-directions, respectively. We consider the normalization condition

$$
\int_{\mathbb{R}^{3}}|\psi(t, x, z)|^{2} d(x, z)=1
$$

For studying the asymptotic behavior as $\varepsilon \rightarrow 0$, we introduce the expansion of the solution $\psi(t, x, z)$ with respect to the eigenstates $r_{k}(z)$ of $H$ :

$$
\psi(t, x, z)=\sum_{k \geq 0} e^{-i \mu_{k} t / \varepsilon^{2}} \phi_{k}(t, x) r_{k}(z),
$$

where $\mu_{k}$ denote the corresponding eigenvalues, i.e. $H r_{k}=\mu_{k} r_{k}, k \geq 0$.

We expect that the modulation wave functions $\phi_{k}$ have strong limits as $\varepsilon \rightarrow 0$. Actually, for initial data belonging to the subband of the ground state energy level $\mu_{0}$ of the dominating harmonic oscillator $H$, it can be proven that in the limit $\varepsilon \rightarrow 0$ the mass remains concentrated in this subband. The modulation wave function satisfies a (lower dimensional) GP-equation [7]. For general initial data, the formal asymptotic limit has been carried out in [3] by the averaging method and later it was rigorously justified in a functional framework in [6]. The limiting equations for the modulation wave functions are given by

$$
i \partial_{t} \phi_{k}=H^{\perp} \phi_{k}+\sum_{\substack{l, m, n \geq 0 \\ \mu_{k}+\mu_{m}-\mu_{l}-\mu_{n}=0}} \gamma_{k l m n} \phi_{l} \overline{\phi_{m}} \phi_{n}, \quad k \geq 0, t \geq 0, x \in \mathbb{R}^{e}
$$

with $\gamma_{k l m n}=\gamma \int_{\mathbb{R}^{d}} r_{k}(z) r_{l}(z) r_{m}(z) r_{n}(z) d z$. This work is concerned with numerical methods for this system. As a first step, we truncate at a finite index $K$ and consider only a finite number of equations:

$$
\begin{aligned}
& i \partial_{t} \phi_{k}=H^{\perp} \phi_{k}+\sum_{\substack{0 \leq l, m, n \leq K \\
\mu_{k}+\mu_{m}-\mu_{l}=\mu_{n}=0}} \gamma_{k l m n} \phi_{l} \overline{\phi_{m}} \phi_{n}, \quad 0 \leq k \leq K \\
& \phi_{k}(t=0, x)=\phi_{k}^{I}(x) .
\end{aligned}
$$


This finite system has been analyzed in [3], where we introduced a numerical discretization method based on a multistep time splitting spectral scheme and designed the numerical method for $K=0, \ldots, 3$. Here we are interested in systems with an arbitrary number of equations $K$, and, since the method presented in [3] is not easy to generalize to bigger $K$, we develop a different discretization based on a Hermite pseudo-spectral method combined with a Crank-Nicolson type method.

The paper is organized as follows. In the following section, properties of the finite system are discussed. Then we specialize to the case $e=1$, i.e., a cigar-shaped condensate. A splitting scheme is presented, with a Hermite pseudo-spectral method for the one-dimensional harmonic oscillator, combined with a Crack-Nicolson type discretization for the ODE system corresponding to the nonlinear coupling. At the end of the section the accuracy and stability of the new method are discussed. In section 3, numerical results are reported and compared to those obtained by the methods in [3].

\section{Numerical approximation}

Before the numerical method is presented, we would like to give a short overview of the properties of the system (1.3). The unique solution $\Phi=\left(\phi_{0}, \phi_{1}, \ldots, \phi_{K}\right)^{T}$ (Theorem 2.1 in [3]) satisfies the following conservation laws:

$$
E_{1}^{K}[\Phi]=E_{1}^{K}\left[\Phi^{I}\right], \quad E_{2}^{K}[\Phi]=E_{2}^{K}\left[\Phi^{I}\right], \quad M^{K}[\Phi]=M^{K}\left[\Phi^{I}\right],
$$

with

$$
\begin{aligned}
E_{1}^{K}[\Phi]: & \left.=\sum_{k=0}^{K} \int_{\mathbb{R}^{e}} \frac{1}{2}\left[\left|\nabla_{x} \phi_{k}\right|^{2}+\left|x \phi_{k}\right|^{2}+f_{k}(\Phi)\right) \overline{\phi_{k}}\right] d x, \\
f_{k}(\Phi): & =\sum_{\substack{0 \leq l, m, n \leq K \\
\mu_{k}+\mu_{m}-\mu_{n}-\mu_{l}=0}} \gamma_{k l m n} \phi_{l} \overline{\phi_{m}} \phi_{n}, \\
E_{2}^{K}[\Phi]: & =\sum_{k=0}^{K} \int_{\mathbb{R}^{e}} \mu_{k}\left|\phi_{k}\right|^{2} d x, \\
M^{K}[\Phi]: & =\sum_{k=0}^{K} \int_{\mathbb{R}^{e}}\left|\phi_{k}\right|^{2} d x .
\end{aligned}
$$

Note that the total energy of the original problem (1.2) can be approximated by $E_{1}^{K}[\Phi]+E_{2}^{K}[\Phi] / \varepsilon^{2}$. In the limit $\varepsilon \rightarrow 0$ we observe that both terms are conserved individually.

We easily see that

$$
\sum_{k=K}^{\infty} \int_{\mathbb{R}}\left|\phi_{k}\right|^{2} d x \leq \frac{E_{2}^{\infty}[\Phi]}{\mu_{K}},
$$

since the sequence of eigenvalues $\left\{\mu_{k}\right\}_{k>0}$ is monotonically increasing and tends to infinity. This motivates the truncation of the system at a finite index.

For notational convenience, the numerical approach will be presented for the onedimensional problem. We propose a time-splitting method, where the following two subproblems are solved for $0 \leq k \leq K$ :

$$
\begin{aligned}
i \partial_{t} \phi_{k} & =H^{\perp} \phi_{k}, \\
i \partial_{t} \phi_{k} & =\sum_{\substack{0 \leq l, m, n \leq K \\
\mu_{k}+\mu_{m}-\mu_{l} \leq \mu_{n}=0}} \gamma_{k l m n} \phi_{l} \overline{\phi_{m}} \phi_{n} .
\end{aligned}
$$


Note that the splitting is different from the one used in [3], where the confinement potential was a part of the second step.

We denote the eigenstates and eigenvalues of $H^{\perp}$ by:

$$
\rho_{n}(x)=h_{n}(x) e^{-x^{2} / 2}, \quad \sigma_{n}=n+1 / 2,
$$

with $n \geq 0$ and $h_{n}(x)$ being the scaled Hermite polynomials [11]:

$$
h_{n}(x)=\frac{1}{\pi^{1 / 4} \sqrt{2^{n} n !}} H_{n}(x) .
$$

For the spatial discretization we take as grid points the scaled Hermite-Gauß points. For quadrature of order $N$ they are given by the zeroes $x_{\iota}$ with $\iota=0, \ldots, N$ of $H_{N+1}(x)$ [11], which lie symmetrically about $x=0$. Let the time step be $h>0$ and $t_{j}:=j h, j \geq 0$. The numerical approximation of $\phi_{k}\left(t_{j}, x_{\iota}\right)$ is denoted by $\phi_{k}^{j, \iota}$,

Given the approximate solution at time $t_{j}$, i.e., $\phi_{k}^{j, 0}, \ldots, \phi_{k}^{j, N}$, we start by solving (2.1). As a first step, these values are interpolated by a linear combination of the first $N+1$ eigenstates of $H^{\perp}$ :

$$
\phi_{k}^{j, \iota}=\sum_{n=0}^{N} a_{k}^{j, n} \rho_{n}\left(x_{\iota}\right), \quad \iota=0, \ldots, N .
$$

For the computation of the Fourier coefficients, we introduce the scaled Hermite-Gauß weights (see $[5,12])$

$$
w_{\iota}=\frac{2^{N} N ! \sqrt{\pi}}{(N+1) H_{N}\left(x_{\iota}\right)^{2}} e^{x_{\iota}^{2}}, \quad \iota=0, \ldots, N .
$$

Then the Fourier coefficients are computed by Gauß-Hermite quadrature [12]:

$$
a_{k}^{j, n}=\sum_{\iota=0}^{N} w_{\iota} \phi_{k}^{j, \iota} \rho_{n}\left(x_{\iota}\right), \quad n=0, \ldots, N .
$$

Now the solution after the first half-step (2.1) is given by

$$
\widehat{\phi_{k}^{j, \iota}}=\sum_{n=0}^{N} a_{k}^{j, n} \rho_{n}\left(x_{\iota}\right) e^{-i \sigma_{n} h} .
$$

Before the discretization of the nonlinear part is introduced, we rewrite $(2.2)$ in vector notation:

$$
i \partial_{t} \Phi=A(\Phi) \Phi
$$

The choice of the matrix $A(\Phi)$ is not unique. For arbitrary $p \in \mathbb{R}$ and with the definition

$$
A(\Phi)_{k n}:=\sum_{\substack{0 \leq l, m \leq K \\
\mu_{k}+\mu_{m}-\mu_{l}-\mu_{n}=0}} \alpha_{k l m n} \gamma_{k l m n} \phi_{l} \overline{\phi_{m}}, \quad \text { with } \alpha_{k l m n}=\left\{\begin{array}{c}
p \quad \text { if } k=n \neq l, \\
2-p \text { if } k=l \neq n, \\
1 \quad \begin{array}{l}
\text { otherwise }
\end{array}
\end{array}\right.
$$

the right hand sides of (2.3) and of (2.2) are the same. The observation that the coefficients $\alpha_{k l m n}$ and $\gamma_{k l m n}$ are symmetric with respect to $(k, l) \leftrightarrow(m, n)$ shows that $A(\Phi)$ is Hermitian. 
As a motivation for the discretization of (2.3), we first consider the nonlinear part of the original equation (1.2):

$$
i \partial_{t} \psi=|\psi|^{2} \psi
$$

Note that the absolute value of $\psi$ is conserved in time by this equation. This implies

$$
\psi\left(t_{j+1}\right)=e^{-i h\left|\psi\left(t_{j}\right)\right|^{2}} \psi\left(t_{j}\right)
$$

Using the rational approximation $e^{i z} \approx \frac{1+i z / 2}{1-i z / 2}$, which is of third order in $z$ and shares with $e^{i z}$ the property $\left|\frac{1+i z / 2}{1-i z / 2}\right|=1$, the solution of (2.4) can be approximated by

$$
\psi^{j+1}=\frac{1-i h / 2\left|\psi^{j}\right|^{2}}{1+i h / 2\left|\psi^{j}\right|^{2}} \psi^{j},
$$

which corresponds to the trapezoidal scheme

$$
i \frac{\psi^{j+1}-\psi^{j}}{h}=\left|\psi^{j}\right|^{2} \frac{\psi^{j+1}+\psi^{j}}{2} .
$$

This is a second order discretization of (2.4) since the density $|\psi|^{2}$ is constant in time for solutions of (2.4). A second order method for (2.2) can be obtained by a two-step procedure, with

$$
\begin{aligned}
i \frac{\Phi^{j+1 / 2, \iota}-\widehat{\Phi^{j, \iota}}}{h / 2} & =A\left(\widehat{\Phi^{j, \iota}}\right) \widehat{\Phi^{j, \iota}}, \\
i \frac{\Phi^{j+1, \iota}-\widehat{\Phi^{j, \iota}}}{h} & =A\left(\Phi^{j+1 / 2, \iota}\right) \frac{\Phi^{j+1, \iota}+\widehat{\Phi^{j, \iota}}}{2} .
\end{aligned}
$$

REMARK 2.1. The computation of $\Phi^{j+1, \iota}$ involves the solution of a linear system with coefficient matrix $I+i \frac{h}{2} A$, which is invertible since $A$ is hermitian.

Note that for different values of $p \in \mathbb{R}$ we have different discretizations, although the original problem (2.2) is not affected by the choice of $p$.

Summarizing, one step of the Strang splitting method written in vector notation is given by:

- Solving (2.1) on a half time step:

$$
\begin{aligned}
\mathbf{a}^{j, n} & =\sum_{\iota=0}^{N} w_{\iota} \Phi^{j, \iota} \rho_{n}\left(x_{\iota}\right), \quad n=0, \ldots, N, \\
\widehat{\Phi^{j, \iota}} & =\sum_{n=0}^{N} \mathbf{a}^{j, n} \rho_{n}\left(x_{\iota}\right) e^{-i \sigma_{n} h / 2},
\end{aligned}
$$

- Secondly, solving (2.2) on a whole time step:

$$
\begin{aligned}
\Phi^{j+1 / 2, \iota} & =\left(I-i \frac{h}{2} A\left(\widehat{\Phi^{j, \iota}}\right)\right) \widehat{\Phi^{j, \iota}} \\
\widehat{\Phi^{j, \iota}} & =\left(I+i \frac{h}{2} A\left(\Phi^{j+1 / 2, \iota}\right)\right)^{-1}\left(I-i \frac{h}{2} A\left(\Phi^{j+1 / 2, \iota}\right)\right) \widehat{\Phi^{j, \iota}}
\end{aligned}
$$


- And finally, solving again (2.1) on a half time step:

$$
\begin{aligned}
& \widehat{\widehat{\mathbf{a}^{j, n}}}=\sum_{\iota=0}^{N} w_{\iota} \widehat{\widehat{\Phi^{j, \iota}}} \rho_{n}\left(x_{\iota}\right), \quad n=0, \ldots, N \\
& \Phi^{j+1, \iota}=\sum_{n=0}^{N} \widehat{\widehat{\mathbf{a}^{j, n}}} \rho_{n}\left(x_{\iota}\right) e^{-i \sigma_{n} h / 2} .
\end{aligned}
$$

Discretization error and stability. The time discretization error comes on one

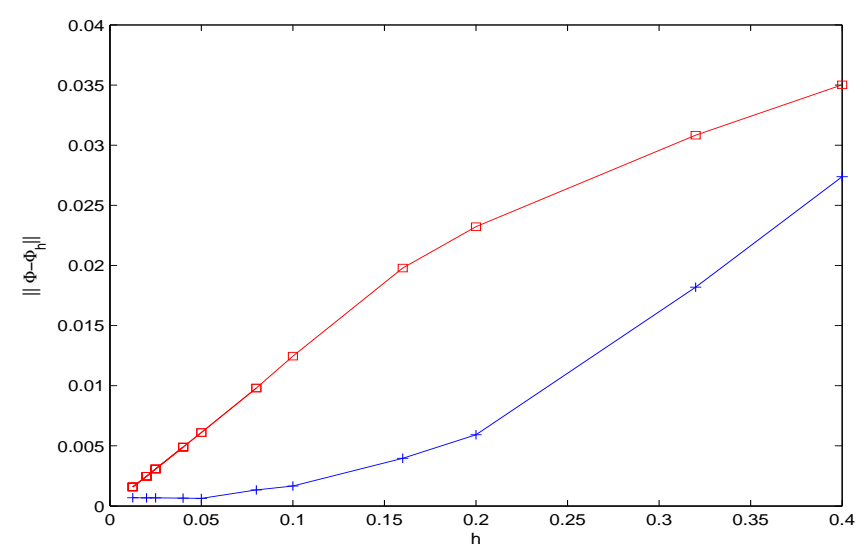

Figure 2.1. Absolute error $\left\|\Phi-\Phi_{h}\right\|$ computed with Strang splitting ('-+') and simple splitting ('- $\square$ ').

hand from the splitting, which is globally of second order in $h$ since we do a Strang splitting. On the other hand, the discretization scheme (2.7) for the nonlinearity is easily shown to be also of second order.

In Fig. 2.1, we plotted the absolute error $\left\|\Phi-\Phi_{h}\right\|$, where we computed the solutions by Strang splitting and by simple splitting. The "exact" $\Phi$ is obtained by solving the problem with the time step $10^{-4}$ and $\Phi_{h}$ by solving it with time step $h$ given in the abscissa. $\|$.$\| denotes the norm \sup _{t}\|g(t, .)\|_{L^{2}(\mathbb{R})}$. We observe in Fig. 2.1 that the simple splitting leads to a first order time discretization error, since the curve has a linear growth for time steps smaller than 0.15. The Strang splitting, on the other hand, leads to a second order time discretization error, since the curve has a super-linear growth.

The introduced method is of spectral accuracy in space. Moreover, it conserves mass.

Lemma 2.1. The Hermite pseudo-spectral method combined with the Crank-Nicolson type method HPSCN (2.9-2.11) is unconditionally stable, since mass is conserved:

$$
\sum_{0 \leq k<K}\left\langle\phi_{k}^{j}, \phi_{k}^{j}\right\rangle=\sum_{0 \leq k<K}\left\langle\phi_{k}^{I}, \phi_{k}^{I}\right\rangle,
$$

where $\langle f, g\rangle:=\sum_{\iota=0}^{N} w_{\iota} f\left(x_{\iota}\right) \overline{g\left(x_{\iota}\right)}$. 
Proof. We first show the conservation of (2.9), thus $\left\langle\phi_{k}^{j}, \phi_{k}^{j}\right\rangle=\left\langle\widehat{\phi_{k}^{j}}, \widehat{\phi_{k}^{j}}\right\rangle$ for all $k<K$. We have:

$$
\begin{aligned}
\left.\widehat{\phi_{k}^{j}} \widehat{\phi_{k}^{j}}\right\rangle & =\left\langle\sum_{m=0}^{N} a_{k, m}^{j} e^{-i \sigma_{m} h} \rho_{m}, \sum_{l=0}^{N} a_{k, l}^{j} e^{-i \sigma_{l} h} \rho_{l}\right\rangle=\sum_{l, m=0}^{N} \overline{a_{k, l}^{j}} a_{k, m}^{j} e^{i\left(\sigma_{l}-\sigma_{m}\right) h} \underbrace{\left\langle\rho_{l}, \rho_{m}\right\rangle}_{\delta_{l m}} \\
& =\sum_{l=0}^{N}\left|a_{k, l}^{j}\right|^{2}=\sum_{l=0}^{N}\left|\left\langle\phi_{k}^{j}, \rho_{l}\right\rangle\right|^{2}=\sum_{l=0}^{N}\left\langle\phi_{k}^{j}, \rho_{l}\right\rangle\left\langle\rho_{l}, \phi_{k}^{j}\right\rangle=\left\langle\phi_{k}^{j}, \phi_{k}^{j}\right\rangle .
\end{aligned}
$$

For the second step (2.11) we want to show $\sum_{0 \leq k<K}\left\langle\widehat{\phi_{k}^{j}}, \widehat{\phi_{k}^{j}}\right\rangle=\sum_{0 \leq k<K}\left\langle\widehat{\widehat{\phi_{k}^{j}}}, \widehat{\phi_{k}^{j}}\right\rangle$. If we use the fact that $A$ is Hermitian we can easily show that

$$
{\widehat{\left(\widehat{\Phi^{j, \iota}}\right.}}^{T} \widehat{\widehat{\Phi^{j, \iota}}}={\overline{\left(\widehat{\Phi}^{j, \iota}\right.}}^{T} \widehat{\Phi^{j, \iota}} .
$$

All in all, we have conservation of the total mass:

$$
\sum_{0 \leq k<K}\left\langle\phi_{k}^{j}, \phi_{k}^{j}\right\rangle=\sum_{0 \leq k<K}\left\langle\phi_{k}^{j+1}, \phi_{k}^{j+1}\right\rangle .
$$

\section{Numerical experiments}

In this section we present some numerical results obtained by the method (HP$\mathrm{SCN}$ ) introduced in the forgoing section.

Example 1. In the first example we analyze the approximation error depending on $\varepsilon$ and $K$, when we approximate the solution $\psi$ of the original problem (1.2) by the solution $\left(\phi_{k}\right)_{0 \leq k<K}$ of the reduced problem (1.3). $\psi$ is solved by the time-splitting spectral method (TSSP) [3, 4] with the parameter values used in [3]. $\left(\phi_{k}\right)_{0 \leq k<K}$ is solved by the Hermite pseudo-spectral method combined with the Crank-Nicolson type method (HPSCN) for the nonlinearity. Since we want to compute the approximation error, we have to evaluate both solutions at the same grid points. This implies that for these experiments we have to use uniform grid points in the space discretization of HPSCN. We choose the interval $[-a, a](a \geq 0)$ such that it contains the support of all Hermite polynomials $\left(H_{k}\right)_{k<N+1}$. Moreover for $N_{x}$ a positive integer we choose the spatial mesh size $h_{x}=2 a / N_{x}$ and let the grid points be:

$$
x_{\iota}=-a+\iota h_{x} \quad \iota=0,1, \ldots N_{x} .
$$

However, for the evaluation of the Fourier coefficients $a_{k, n}^{j}$ we use the trapezoidal quadrature. The discretization error in space is then of second order. In our computation the parameters have the following values: $a=16, N=64$.

Denote the approximation error by $\sigma_{K}^{\varepsilon}(t)$ :

$$
\sigma_{K}^{\varepsilon}(t)=\left\|\psi(., ., t)-\sum_{0 \leq k<K} \phi_{k}(., t) r_{k}(.) e^{-i \mu_{k} t / \varepsilon^{2}}\right\|_{L^{2}(\mathbb{R})} .
$$

where $\psi$ is the solution of (1.2) with initial data $\psi^{I}(x, z)=\sum_{0 \leq k<K} \phi_{k}^{I}(x) r_{k}(z)$ and $\phi_{k}^{I}(x)$ the first eigen states of the harmonic oscillator in $x$, respectively. 


\begin{tabular}{c|c|c}
\hline$\varepsilon$ & $N_{x}$ & $h$ \\
\hline 0.8 & 256 & $10^{-3}$ \\
0.4 & 256 & $10^{-3}$ \\
0.2 & 512 & $10^{-4}$
\end{tabular}

TABLE 3.1. Values for the parameters used in example 1.

\begin{tabular}{c|c|c|c}
\hline$\varepsilon$ & $h$ & $\begin{array}{c}\text { MSTSS } \\
\text { hh:mm:ss }\end{array}$ & $\begin{array}{c}\text { HPSCN method } \\
\text { hh:mm:ss }\end{array}$ \\
\hline 0.8 & $10^{-3}$ & $00: 01: 40$ & $00: 01: 42$ \\
0.4 & $10^{-3}$ & $00: 05: 15$ & $00: 01: 38$ \\
0.2 & $10^{-4}$ & $01: 19: 12$ & $00: 28: 34$
\end{tabular}

TABLE 3.2. Running times of the different methods during computation of the approximation error for $K=3$.

In Table 3.2 we compare the running times for the two methods during the computation of Example 1. The parameters used are those of Table 3.1.

In Fig. 3.1 we see the approximation error for decreasing $\varepsilon$ and increasing $K$. Here, we computed additionally to [3] the approximation error for $K=4$ and $K=5$. However, we can draw the same conclusions as in [3].

REMARK 3.1. During the numerical experiments it turned out that the parameter $p$ has little impact on the results. Therefore, we make the choice $p=2$. This value is moreover the appropriate one for comparisons with the numerical method presented in [3].

In the following experiments the grid points are the roots of the Hermite polynomial $H_{N+1}(x)$. We choose $N=46$, which means that we have 47 grid points on the $x$-axis. The parameter $p$ of the discretization is chosen equal to 2. All results obtained by HPSCN are finally compared to the corresponding quantities computed by the multistep time-splitting scheme presented in [3], which we call MSTSS. In order to achieve similar quality of the data we use for MSTSS 257 uniform grid points on the interval $[-16,16]$. Moreover, we choose the time step $h=0.001$. The initial data are chosen as the first $K$ eigenstates of the operator $-\frac{1}{2} \partial_{x x}+\frac{\gamma_{I}^{2}}{2} x^{2}$, whereby the sum of their masses is normalized to one. The value of $\gamma_{I}$ will be specified individually in every example.

Example 2. In this example we compare the condensate widths as functions of time computed by the two different numerical methods. Let $\left(\tau_{k}\right)_{0 \leq k<K}$ be the condensate width of the corresponding wave function $\phi_{k}$ :

$$
\tau_{k}=\sqrt{\left\langle(x-\langle x\rangle)^{2}\right\rangle} \text { where }\langle f\rangle:=\int_{\mathbb{R}} f(x)\left|\phi_{k}\right|^{2} d x \text { with } 0 \leq k<K .
$$

Here we solve the system for $K=3$, thus $0 \leq k<3$. Moreover, $\gamma_{I}=10$.

Example 3. Here we compute the mass for every component of the solution, namely $\left\|\phi_{k}\right\|_{2}^{2}=\int_{\mathbb{R}}\left|\phi_{k}(x)\right|^{2} d x$, with $0 \leq k<k$, with $K=3$. For the initial data $\gamma_{I}=1$. We know that the total mass of the system is conserved. This experiment indicates that there is mass exchange between the different states $\left(\phi_{k}\right)_{0 \leq k<3}$. (See Figure 3.3). 
a.)

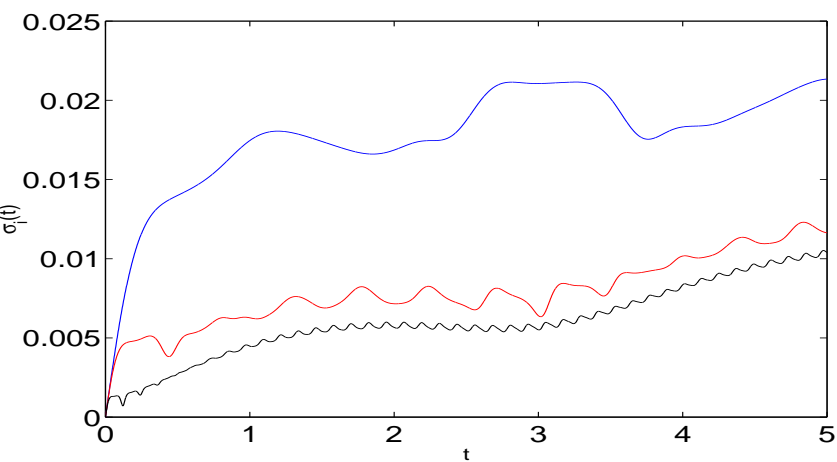

b.)

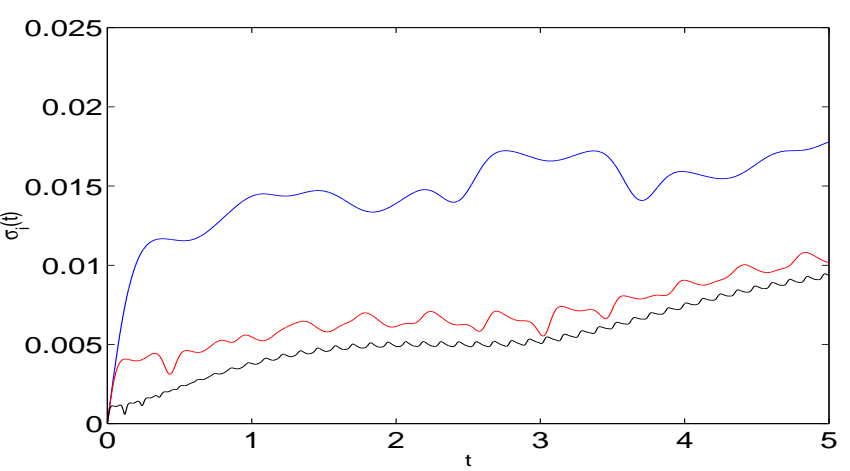

c.)

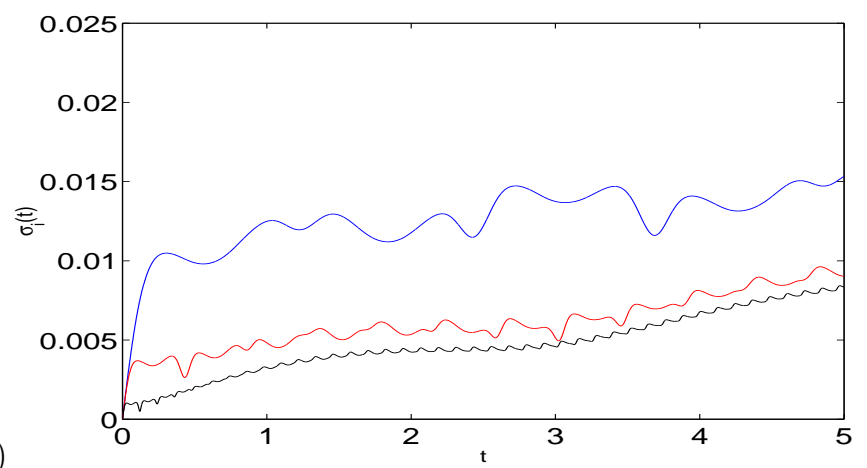

FIGURE 3.1. $\sigma_{K}^{\varepsilon}(t)$ with $\varepsilon=0.8,0.4$ and 0.2 (in the order of decreasing peaks) and a.) $K=3$, b.) $K=4$, c.) $K=5$.

Example 4. Here we observe the evolution in time of the central densities for every component of the solution, namely $\left|\phi_{k}(t, 0)\right|^{2}$, with $0 \leq k<K$, with $K=3$. For the initial data $\gamma_{I}=10$ (see Figure 3.4).

The examples (1-4) show that both methods give similar results. However, comparison of the running time in Table 3.2 highlights the fact that HPSCN is faster than MSTSS. Another advantage of the new method HPSCN is the fact that we can implement it for a greater number of equations $K$, which is rather cumbersome for the MSTSS method.

REMARK 3.2. The representation of the position densities for a greater number of 
a.)
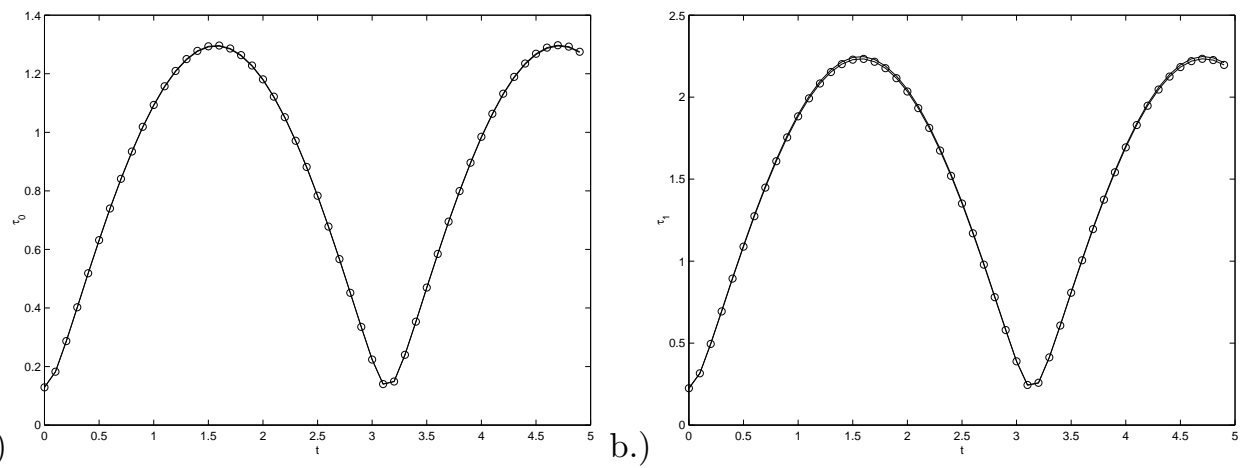

c.)

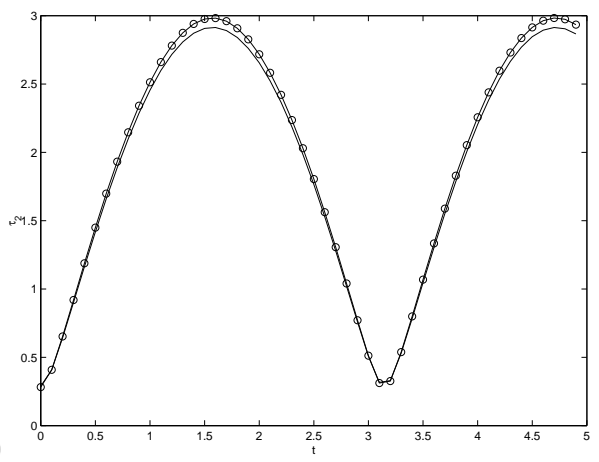

FIGURE 3.2. Condensate widths $\tau_{k}$ as functions of time. The solution $\left(\phi_{k}\right)_{k<3}$ is obtained by HPSCN ('-o') with $p=2$ and 47 grid points and by MSTSS ('-') with 257 grid points on the interval $[-16,16]$. a.) $k=0$, b.) $k=1$, c.) $k=2$.

equations indicates that the solutions may go beyond the interval in which lie the roots of the chosen Hermite polynomial $H_{47}$. Therefore, if we increase $K$, we have to increase $N$, too.

Example 5. Here we want to display the surface plots of the position densities $\left|\phi_{k}(t, x)\right|, \quad 0 \leq k<K$ with $K=5$ and $N=64$. For the initial data we have $\gamma_{I}=10$ and observe the solutions up to $t \leq 5$.

The analysis of Figure 3.5 allows us to assert that the number of peaks increases with $K$. This is an expected behavior since initially the $k$-th excited state has $k+1$ peaks (the ground state has one peak, the first excited state has two peaks etc). In addition, the greater the confining potential, the steeper are the peaks. Last but not least, we observe periodic behavior of the solutions, which is not surprizing in view of Duhamel's formula for nonlinear Schrödinger equations $[8,9]$. Not so evident is the fact that the period length is the same for all states.

\section{Conclusion}

We developed an unconditionally stable, second order accurate in time and spectrally accurate in space method for solving finite systems of Gross-Pitaevskii equations. The method is based on a Hermite pseudo-spectral method combined with a Crank-Nicolson type method, which we called HPSCN. We compared the new method to an existing one (MSTSS) presented in [3] and observed similar results. The advantage of the new method is the easy generalization to a larger number of equations in 
a.)

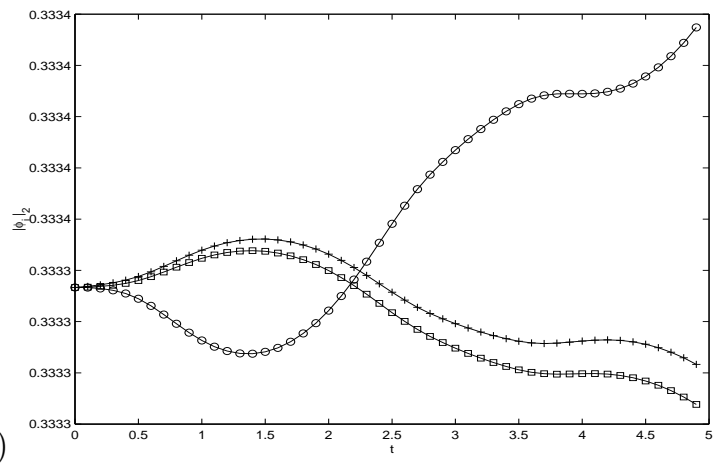

b.)

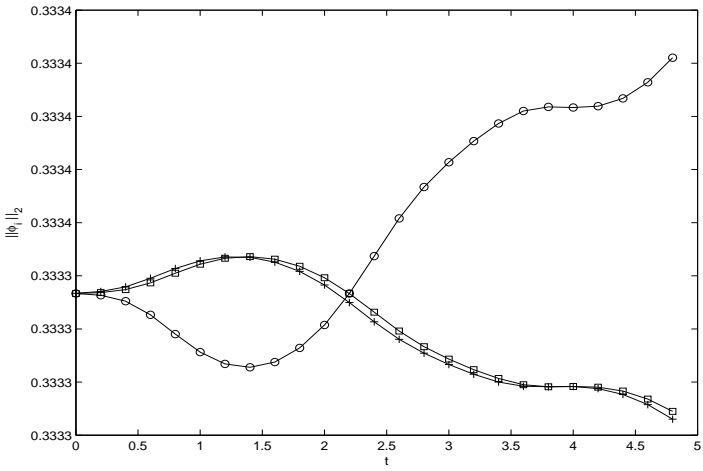

Figure 3.3. Mass $\left\|\phi_{k}\right\|_{2}$ as function of time, $k=0$ ('- $\square$ '), $k=1$ ('-o') and $k=2$ ('-+'). The solution is obtained in a.) by HPSCN with $p=2$ and 47 grid points and in b.) by MSTSS with 257 grid points on the interval $[-16,16]$.

the system. Moreover HPSCN needs fewer grid points than MSTSS for similar quality of data. However, we have to keep in mind that HPSCN is based on the spectral decomposition of the harmonic oscillator; therefore the method works only in the case that we have an harmonic external potential. For another external potential one has to use MSTSS.

Acknowledgements. Support by the PhD program "Differential Equations" funded by the Austrian Science Fund, project no. W8 is acknowledged. Moreover the work has been partly supported by the "Wittgenstein 2000" Award of P. Markowich. The work was supported by the "ALPHA"-Project "PDEs in Industry and Engineering".

\section{REFERENCES}

[1] M. H. Anderson, J. R. Ensher, M. R. Matthews, C. E. Wieman and E. A. Cornell, Observation of Bose-Einstein condensation in a dilute atomic vapor, Science, 269, 198-201, 1995.

[2] J. R Anglin and W. Ketterle, Bose-Einstein condensation of atomic gases, Nature, 416, 211218, 2002

[3] W. Bao, P. A. Markowich, Ch. Schmeiser and R. M. Weishäupl, On the Gross-Pitaevskii equation with strongly anisotropic confinement, M3AS, 15(5), 767-782, 2005.

[4] W. Bao, D. Jaksch and P. A. Markowich, Numerical solution of the Gross-Pitaevskii equation for Bose-Einstein condensation, J. Comput. Phys., 187, 318-342, 2003. 


\section{HERMITE PSEUDO-SPECTRAL METHOD FOR GROSS-PITAEVSKII EQUATIONS}

a.)

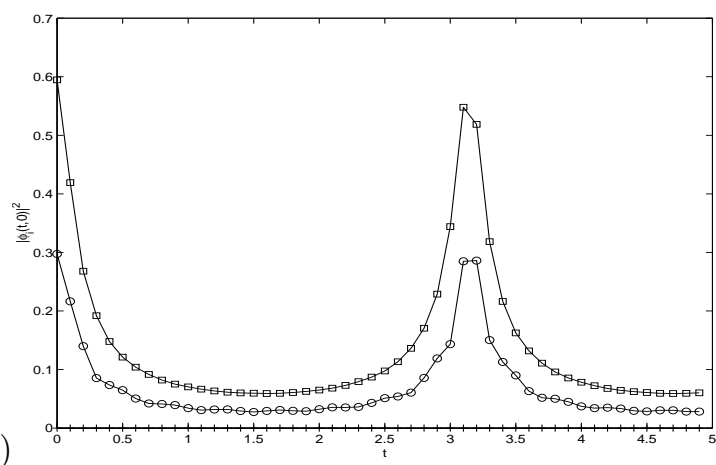

b.)

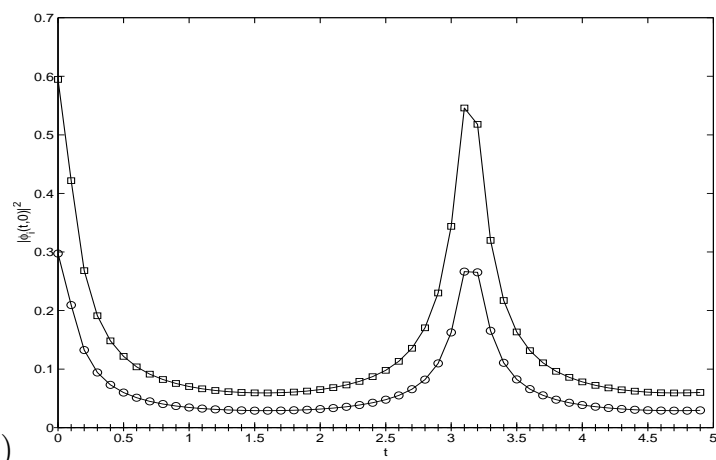

Figure 3.4. Central density $\left|\phi_{k}(t, 0)\right|^{2}$ as function of time, $k=0$ ('- $\square$ '), $k=1$ ('-+') and $k=2$ ('-o'). The solution is obtained in a.) by HPSCN with $p=2$ and $47(N=46)$ grid points and in b.) by MSTSS with 257 grid points on the interval $[-16,16]$.

[5] W. Bao and J. Shen, A fourth-order time-splitting Laguerre-Hermite pseudo-spectral method for Bose-Einstein condensates, SIAM J. Sci. Comput, 26(6), 2010-2028, 2005.

[6] N. Ben Abdallah, F. Méhats and F. Castella, Time averaging for the strongly confined nonlinear Schrödinger equation, preprint.

[7] N. Ben Abdallah, F. Méhats, Ch. Schmeiser and R. Weishäupl, The nonlinear Schrödinger equation with a strongly anisotropic harmonic potential, SIAM J. Math. Anal, 37(1), 189199, 2005

[8] R. Carles, Remarks on nonlinear Schrödinger equations with harmonic potential, Ann. Henri Poincaré, 3, 757-772, 2002.

[9] T. Cazenave, Semilinear Schrödinger Equations, Courant Lect. Notes Math. 10, AMS, Providence, RI, 2003.

[10] F. Dalfovo, S. Giorgini, L. P. Pitaevskii and S. Stringari, Theory of Bose-Einstein condensation in trapped gases, Rev. Mod. Phys., 71(3), 463-512, 1999.

[11] R. Dautray and J.-L. Lions, Mathematical Analysis and Numerical Methods for Science and Technology, Springer-Verlag, 5, 2000.

[12] D. Funaro, Polynomial Approximations of Differential Equations, Springer-Verlag, 1992.

[13] A. Görlitz, J. M. Vogels, A. E. Leanhardt, C. Raman, T. L. Gustavson, J. R. Abo-Shaeer, A. P. Chikkatur, S. Gupta, S. Inouye, T. Rosenband and W. Ketterle, Realization of BoseEinstein condensate in lower dimensions, Phys. Rev. Lett., 87, 130402-4, 2001.

[14] E. H. Lieb, R. Seiringer and J. Yngvason, One-dimensional behaviour of dilute, trapped Bose gases, Comm. Math. Phys., 244(2), 347-393, 2004.

[15] V. M. Pérez-García and X. Liu, Numerical methods for the simulation of trapped nonlinear Schrödinger systems, Appl. Math. and Comp., 144, 215-235, 2003.

[16] L. Pitaevskii and S. Stringari, Bose-Einstein Condensation, Oxford Science Publications, 2003. 
a.)

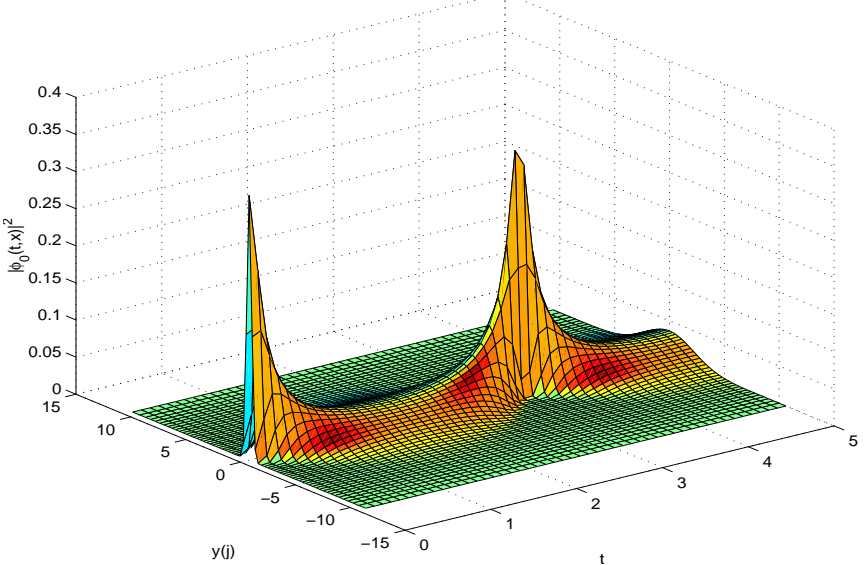

b.)

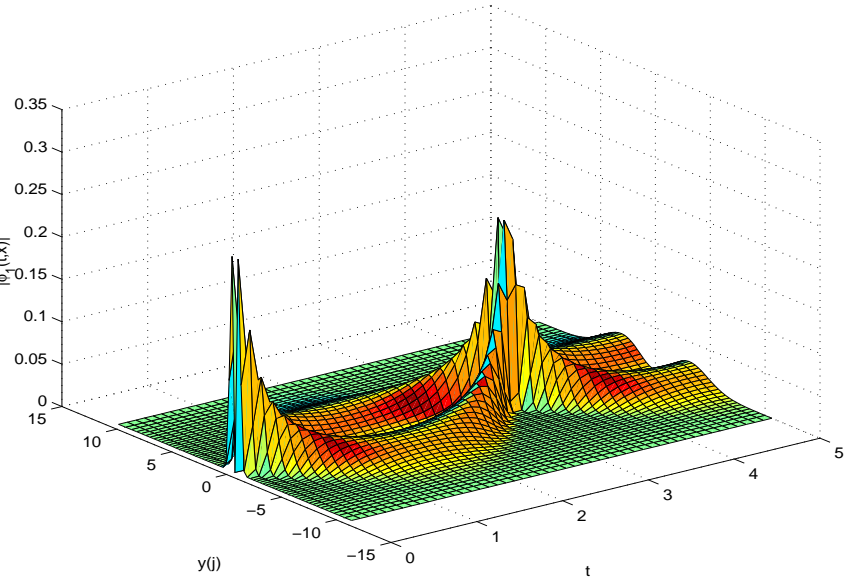

c.)

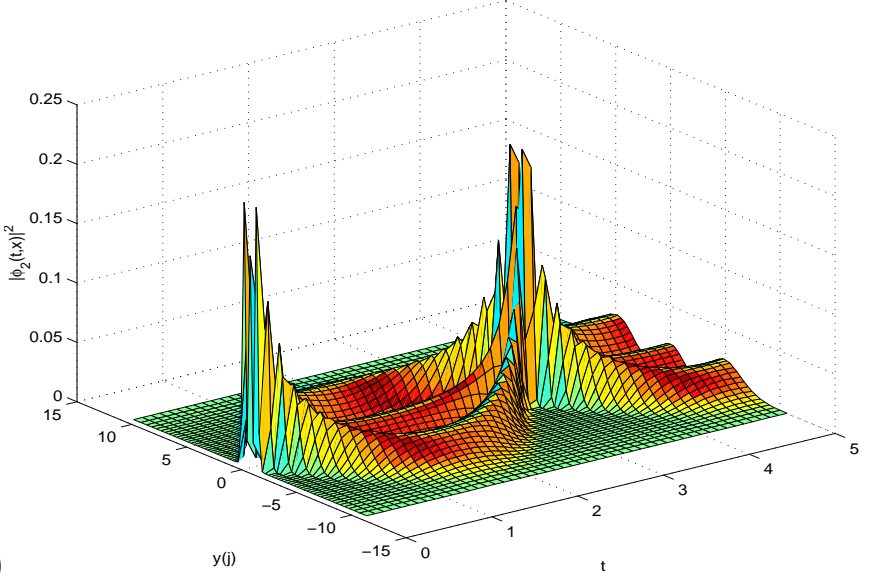


d.)

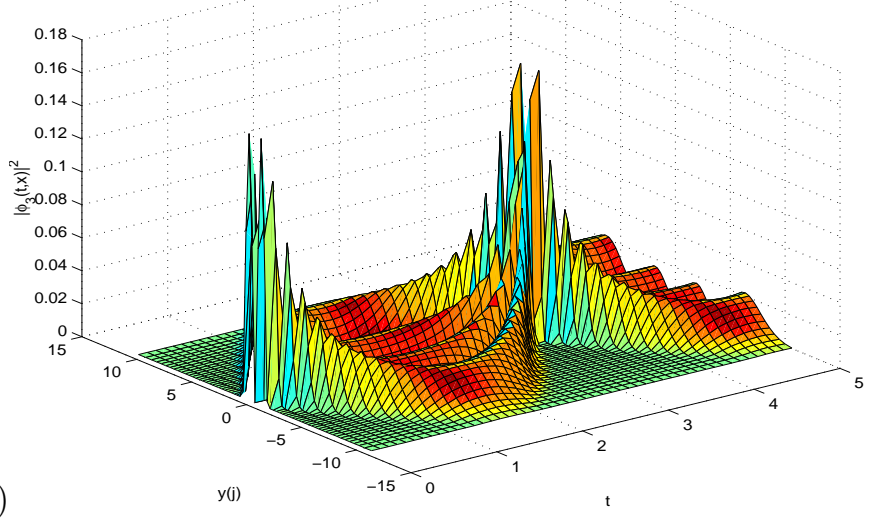

e.)

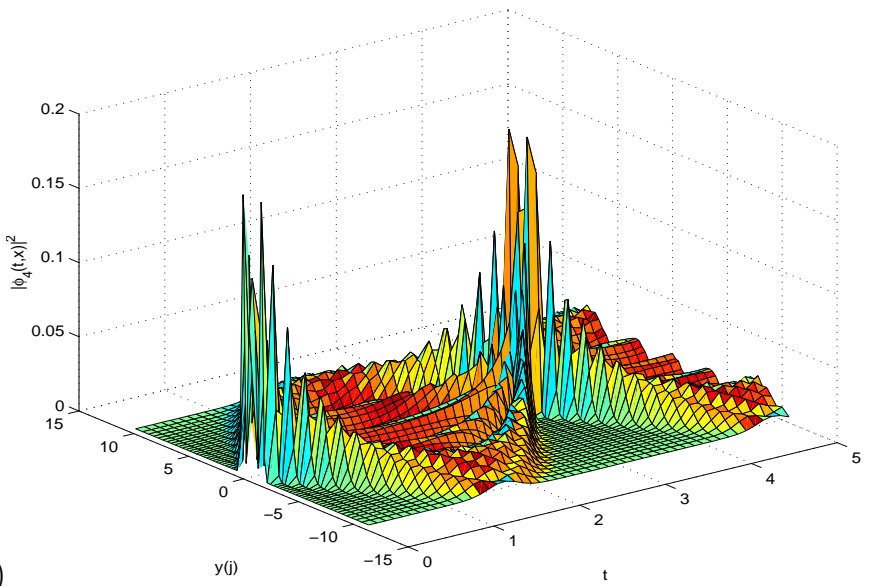

Figure 3.5. Position densities $\left|\phi_{k}(t, x)\right|$ for $0 \leq k<5$ 